\title{
LARGE TIME BEHAVIOR OF THE HEAT KERNEL: ON A THEOREM OF CHAVEL AND KARP
}

\author{
BARRY SIMON
}

(Communicated by Palle E. T. Jorgensen)

\begin{abstract}
We show that a theorem of Chavel and Karp follows from the spectral theorem and elliptic regularity
\end{abstract}

Recently Chavel and Karp [1] proved the following:

Let $M$ be a noncompact Riemannian manifold with Laplace-Beltrami operator $\Delta$ acting on functions on $M, \lambda=: \lambda(M)$ the bottom of $\operatorname{spec}(-\Delta)$, and attendant minimal positive heat kernel $p(x, y, t)$ (where $(x, y, t)$ is an element of $M \times M \times(0,+\infty))$.

Theorem. For all $x, y$ in $M$ we have the existence of the limit

$$
\lim _{t \uparrow+\infty} e^{\lambda t} p(x, y, t)=: \mathscr{F}(x, y),
$$

for which we have the following alternative:

Either vanishes identically on all of $M \times M$, in which case $\lambda$ possesses no $L^{2}$ eigenfunctions, or $\mathscr{F}$ is strictly positive on all of $M \times M$, in which case $\lambda$ possesses a positive normalized $L^{2}$ eigenfunction $\phi$ (normalized in the sense that its $L^{2}$ norm is equal to 1) for which

$$
\lim _{t \uparrow+\infty} e^{\lambda t} p(x, y, t)=\phi(x) \phi(y)
$$

locally uniformly on all of $M \times M$.

Our goal here is to show that this result is essentially an immediate consequence of the spectral theorem and elliptic regularity.

The following well-known lemma follows directly form the spectral theorem and the Lebesgue monotone convergence theorem.

Lemma. Let $A$ be a selfadjoint operator and let $f(x, t)$ be a measurable function on $\sigma(A) \times[0, \infty]$ so that $f(x, \cdot)$ is monotone for each fixed $x$ and $f(x, \infty)$ $=\inf _{t} f(x, t)=\lim _{t} f(x, t)$. Then, $s-\lim _{t \rightarrow \infty} f(A, t)=f(A, \infty)$.

For $t<\infty$, let $f(x, t)=e^{-t(x-\lambda)}$ and let $f(x, \infty)=\delta_{\lambda}(x)$, the characteristic function of $\{\lambda\}$. Then $f(-\Delta, \infty)$ is the projection $P$ onto the space $S$ of

Received by the editors October 5, 1991.

1991 Mathematics Subject Classification. Primary 35K05, 53C99.

Research partially supported by USNSF under grant number DMS-9101715. 
all $L^{2}$ eigenfunctions with eigenvalue $\lambda$. Since $p(x, y, t)$ is strictly positive, the Perron-Frobenius theorem (see [2, §XIII.12]) implies that either $S=\{0\}$ or is one-dimensional with a unique element $\varphi$ so that $\varphi(x)>0$ and $\|\varphi\|_{2}=1$. Thus, either $f(-\Delta, \infty)=0$ or $f(-\Delta, \infty)=(\varphi, \cdot) \varphi$ as operators.

Equation (1) therefore holds from the lemma if convergence is intended in the $L^{2}$ sense. To turn this into pointwise convergence (even local $C^{\infty}$ ), we need only appeal to elliptic regularity.

By elliptic regularity, $C^{\infty}(H) \equiv \bigcap_{n} D\left(\Delta^{n}\right) \supset \operatorname{Ran}\left(e^{t \Delta}\right)$ consists of $C^{\infty}$ functions. Thus, $f \mapsto\left(e^{+\Delta} f\right)(x)$ is a bounded functional on $L^{2}$. By duality $g_{x}(y) \equiv\left(e^{(\Delta+\lambda)}\right)(x, y)$ is in $L^{2}$. Thus, by the strong $L^{2}$ convergence and the semigroup property,

$$
e^{\lambda(t+2)} p(x, y, t+2)=\int g_{x}(z) e^{\lambda t} p(z, w, t) g_{y}(w) d t d w
$$

converges to $\left(g_{x}, P g_{y}\right)=P(x, y)$. This proves the theorem.

We close with several remarks:

1. Since elliptic regularity implies that $C^{\infty}(H)$ consists of $C^{\infty}$ functions, it is not hard to see that the convergence is in the $C^{\infty}$ topology.

2. We did not provide a proof of the last statement in the main theorem of [1] that $\lim _{x \rightarrow \infty} \varphi(x)=0$ if $M$ is noncompact Riemannian with bounded geometry. This should follow by a general subsolution estimate that bounded geometry implies that

$$
|\varphi(x)| \leq c \int_{\rho(x, y) \leq 1}|\varphi(y)| d y .
$$

3. By the proof, the operators $A_{t}=e^{\lambda t} e^{\Delta t}$ are monotone decreasing in $t$. This implies that $\left(\delta_{x}, A \delta_{x}\right)=A(x, x)$ is monotone as noted by Chavel-Karp but also that $A(x, x)+A(y, y) \pm 2 A(x, y)=\left(\delta_{x} \pm \delta_{y}, A\left(\delta_{x} \pm \delta_{y}\right)\right)$ is monotone, providing a direct proof of pointwise convergence.

\section{REFERENCES}

1. I. Chavel and L. Karp, Large time behavior of the heat kernel: the parabolic $\lambda$-potential alternative, Comment. Math. Helv. 66 (1991), 541-556.

2. M. Reed and B. Simon, Methods of modern mathematical physics IV. Analysis of operators, Academic Press, London, 1978.

Division of Physics, Mathematics and Astronomy, 253-37, California Institute of Technology, Pasadena, California 91125

E-mail address: bsimon@caltech.edu 